NASA-CR-204726

\title{
On the retrieval of lightning radio sources from time-of-arrival data
}

\author{
William J. Koshak \\ Space Sciences Laboratory, NASA Marshall Space Flight Center. Huntsville, Alabama
}

\section{Richard J. Solakiewicz}

Department of Mathematics and Computer Science, Chicago State University, Chicago, Illinois

\begin{abstract}
We examine the problem of retrieving three-dimensional lightning locations from radio frequency time-of-arrival (TOA) measurements. Arbitrary antenna locations are considered. By judiciously differencing measurements that are related to the location of the antennas and their excitation times, the problem is converted from the initial spherical nonlinear form to a system of linear equations. In the linear formalism, the source location and time-of-occurrence is viewed geometrically as an intersection of hyperplanes in the four-dimensional Minkowski space $(x, y, z, t)$. The linear equations are solved to obtain explicit analytic expressions for the location and time variables. Retrieval errors are not interpreted with conventional Geometrical Dilution of Precision (GDOP) arguments as discussed by Holmes and Reedy [1951], but with more recent inversion analyses considered by Twomey [1977]. Measurement errors are propagated analytically so that the specific effect of these errors on the solution is clarified. The sensitivity of the solution on the number of antennas used, antenna network geometry, source posi tion, and measurement differencing schemes are discussed in terms of the eigenvalues of the linear system.
\end{abstract}

\section{Introduction}

A variety of data analysis techniques and hardware have been used in the retrieval of lightning locations from ground-based radio frequency time-of-arrival (TOA) measurements [Holmes and Reedy, 1951; Lewis et al., 1960; Oetzel and Pierce, 1969; Proctor, 1971; Cianos et al., 1972; Murty and MacClement, 1973; MacClement and Murty, 1978; Taylor. 1978; Rustan et al., 1980; Bent et al., 1983; Thomson et al., 1994; and Hager and Wang, 1995]. Primary differences between these studies include the number and type of antennas used, the antenna baseline, whether a two-dimensional or three-dimensional fix of the source is desired, the mathematical means for retrieving source location, and the procedure for estimating retrieval error.

The radio source location in the $x y$ plane has been considered, in the mathematical sense, as an intersection of hyperbolas. Given two distinct antenna locations, an arbitrary radio point source will excite each antenna at a specific time. If the positive difference in these excitation times is multiplied by the wave propagation speed, the resulting distance value will define a hyperbola in the $x y$ plane with foci given by the two antenna locations. (Note that this follows directly from the definition of a hyperbola: The set of points such that the positive difference of the distances from two fixed points (called foci) to any point in the set is the same for all points in the set.) In the absence of measurement errors, the set of points $(x, y)$ defining the hyperbola represents the solution space of possible locations of the radio

Copyright 1996 by the American Geophysical Union.

Paper number $96 \mathrm{JD} 01618$

0148-0227/96/96.JD-01618\$09.00 source. One branch of this hyperbola can be excluded from the solution space by examining which antenna was excited first by the source.

Since two antennas cannot uniquely specify the source location in the $x y$ plane, a third antenna is used. That is, two distinct hyperbola branches are defined by considering two possible pairs of the three antennas. The source is located where these hyperbola branches intersect.

Unfortunately, it is possible for two hyperbola branches to intersect at two locations. In this case a fourth antenna can be used to remove source location ambiguity. In addition, modified hyperbolic equation sets are often considered when it is desired to fix the source on the spheroidal Earth. The reader is refered to Holle and Lopez [1993] for further comments and illustrations on two-dimensional fixing of lightning radiation sources as they pertain to the Lightning Position and Tracking System (LPATS).

The more formidable task of determining the threedimensional source location $(x, y, z)$ can be viewed as the mathematical intersection of hyperboloids of two sheets. The appendix of Proctor [1971] discusses some aspects of this problem; it describes a nonlinear numerical solution that involved iterative improvements of an initial source location estimate. An analytic solution with analytic error results (generalized to arbitrary antenna network geometries and source location) was not obtained.

In a recent paper by Thomson et al., [1994] a method was introduced as a variation to Proctor's approach called a "weighted hyperbolic technique." In addition, a variety of improvements in analysis software, hardware (specifically timing accuracy), and calibration techniques were implemented in this five-antenna TOA system. Solution retrieval errors were studied 
using nonlinear numerical methods, and general analytic expressions for retrieval errors were not investigated.

The clever investigation by Taylor [1978] avoids hyperbolic equations altogether by deducing the azimuth and elevation angles from a system of three ground-based antennas and one elevated antenna. Using two of these four-antenna network systems, simple triangulation is used to locate the source in $(x, y, z)$. An obvious drawback of this approach is the need for eight antennas in the total system.

A retrieval algorithm based on the early work of Holmes and Reedy [1951] is currently employed in the Lightning Detection and Ranging (LDAR) system located at the NASA Kennedy Space Center, Florida. This system is described by Poehler and Lennon [1979] and a performance analysis is provided by both Poehler [1977] and Maier et al. [1995].

The study by Holmes and Reedy [1951] identifies certain aspects of the effects of antenna network placement, measurement errors, and source location on final retrieval errors. This is accomplished by a Geometrical Dilution of Precision (GDOP) analysis that has been used by Maier et al. [1995] to assess LDAR retrieval errors.

In this work, we describe a general analytic solution to the problem of determining the three-dimensional location and timeof-occurrence of lightning radio sources from a set of four or more (arbitrarily located) electric field antennas, when measurement errors are present. By judiciously combining certain measurements we eliminate the nonlinear space and time variables inherent in the transit equations that define the excitation time of each antenna. We emphasize the proper mathematical interpretation of the solution space, describing it as an intersection of hyperplanes in the four-dimensional Minkowski space $(x, y, z, t)$. An analytic expression is also derived that clarifies the effect of antenna network geometry, time-differencing schemes, source location, and measurement error on the final solution retrieval error. Unlike earlier GDOP error analyses [Holmes and Reedy, 1951], we apply the more recent linear inversion theory by Twomey [1977] to clarify and emphasize the underlying nature of retrieval errors in this problem. The relationship between measurement error (that is, timing and antenna placement errors), the eigenvalues of the linear system, and error magnification are stressed. Several computer-simulated tests of the retrieval method are provided and we conclude with some comments on how to improve/optimize processing of LDAR data.

\section{Method}

The development in this section was presented by Koshak and Christian [1994]. A similar development is provided by Hager and Wang [1995]. Figure 1 depicts a radio lightning (point) source a distance $R_{i}$ from the $i^{t h}$ antenna. The $i^{t h}$ antenna is excited at time, $t_{i}$, given by

$$
t_{i}=t+R_{i} / v \text {. }
$$

Here, $t$ is the source activation time, $R_{i} / v$ the transit time of the radio wave, and $v$ is the speed of light in air. The direction of the relative position vector, $\mathbf{R}_{i}$, follows standard physics convention, that is, it is directed from the source to the observation point. Note that additional known time-delays (e.g., cable time-delays) can be added to this expression if necessary without affecting the essential mathematical forms to follow. Note also that we have neglected curved transit paths due to refractive effects in the atmosphere.

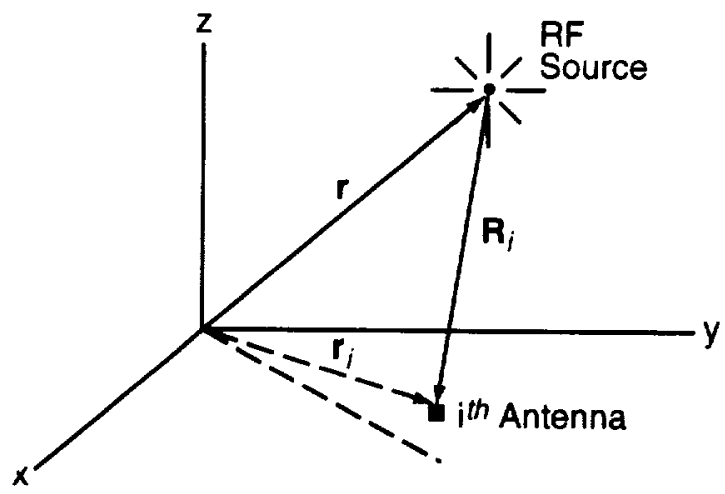

Figure 1. The geometry for lightning radio source retrieval.

Solving (1) for $R_{i}$, squaring, and grouping all terms nonlinear in the source range, $r$, and activation time, $t$, leads to the following form, $a_{i}$, defined by

$a_{i} \equiv\left(r_{i}^{2}-v^{2} t_{i}^{2}\right) / 2=\left(x_{i} x+y_{i} y+z_{i} z-v^{2} t_{i} t\right)-\left(r^{2}-v^{2} t^{2}\right) / 2$

We now define a new measurement, $g_{i j}$, that is proportional only to the linear variables $(x, y, z, t)$. This is achieved by considering the $i^{\text {th }}$ and $j^{\text {th }}$ antennas, and constructing the difference

$$
\begin{aligned}
g_{i j} & \equiv a_{i}-a_{j}=\left(x_{i}-x_{j}\right) x+\left(y_{i}-y_{j}\right) y \\
& +\left(z_{i}-z_{j}\right) z-v^{2}\left(t_{i}-t_{j}\right) t
\end{aligned}
$$

For $n \geq 4$ antennas labeled $1,2, \ldots, n$ there are $p$ possible constraint equations of the form given in (3), where $p$ is a combination of $n$ antennas taken 2 at a time [i.e., $p=C_{n, 2}=n(n-1) / 2$ ]. Of the $p$ equations, only $m=n-1$ are linearly independent. In this study, we consider only the $m$ independent equations given by,

$$
\mathbf{g}=\mathbf{K} \mathbf{f}=\operatorname{col}\left(g_{21}, g_{31}, \ldots, g_{m \mathbf{l}}\right),
$$

where $\mathbf{f}=\operatorname{col}\left(x, y, z, \rightarrow^{2} t\right)$, and $\mathbf{K}$ is a $(m \times 4)$ matrix given by

$$
\mathbf{K}=\left[\begin{array}{cccc}
x_{21} & y_{21} & z_{21} & t_{21} \\
x_{31} & y_{31} & z_{31} & t_{31} \\
\vdots & \vdots & \vdots & \vdots \\
x_{m 1} & y_{m 1} & z_{m 1} & t_{m 1}
\end{array}\right]
$$

We have invoked the differencing notation $\alpha_{i j} \equiv \alpha_{i}-\alpha_{j}, \alpha=$ $x, y, z$, or $t$, for brevity. Other differencing schemes are possible, that is, all differences could be taken with respect to antenna number 2 for example. Some differencing schemes can be shown to be more optimum than others from the standpoint of the degree of error magnification that results for a particular source location (see section 4 below). Note that the linear system in (4) can also be taken as an under-or over-determined system of equations that can be solved using the general theory of constrained linear inversion [see Twomey, 1977; chapter 6].

Finally, note that the $\mathbf{K}$-matrix conveniently summarizes all spatial and temporal difference measurements between the various antennas, and analytic forms for $\mathbf{K}^{-1}$ are obtainable. The time-difference matrix, $\mathbf{T}$, with elements $t_{i j}$ is traceless, antisymmetric, and obeys the useful addition property given by 


$$
t_{i j}=t_{i k}+t_{k j}
$$

This result will be used to simplify a particular form of $\mathbf{K}^{-1}$ associated with a triangular array of antennas (see section 7 below).

\section{Note on Terminology}

In the literature, the usage of the phrase "hyperbolic system" is frequently used to describe the multiple station TOA antenna network. This terminology originates from the consideration of simple two-dimensional hyperbola branch intersections that define radio source location (see section 1). Unfortunately, when one considers three-dimensional source location problems direct intersection of hyperboloidal surfaces has been found to be difficult [Proctor, 1971; appendix A]. However, mention of hyperboloidal surfaces is not even required to solve the problem.

The form given in (1) can be written: $R_{i}^{2}=v^{2}\left(t_{i}-t\right)^{2}$, which defines a spherical surface in position space $(x, y, z)$ since $t$ is regarded as a constant. The center of the sphere is located at the $i^{\text {th }}$ antenna $\left(x_{i}, y_{i}, z_{i}\right)$ and has radius $v\left|t_{i}-t\right|$. Because the light ning source must be located on or above the $x y$ plane, that portion of the spherical solution space lying below the $x y$ plane is excluded.

The spherical form was removed by using the derived difference measurement given in (3). The form of (3) is that of a hyperplane in the four-dimensional space $(x, y, z, t)$, or Minkowski space. Hence, the lightning radio source location and time-of-occurrence is most concisely defined as the intersection of four hyperplanes in Minkowski space. In this sense, the nomenclature "hyperplane system" is more appropriate than "hyperbolic system."

\section{Analytic Expression for Retrieval Errors}

We now discuss the errors that arise when one attempts to solve (4). Unlike the approximative nonlinear error analyses discussed by Holmes and Reedy [1951], Proctor [1971], and Thomson [1994], our error results are straightforward, linear, and analytic.

We start with a linear system of the form given in (4)

$$
\mathbf{g}^{\prime}=\mathbf{K}^{\prime} \mathbf{f}^{\prime} \text {, }
$$

where primed variables indicate exact quantities, that is, none of these variables are associated with measurement errors. If the actual location of the $i^{\text {th }}$ antenna, $\mathbf{r}_{i}^{\prime}$, is measured with location error, $\mathbf{e}_{i}$, and if the actual TOA difference between the $i^{\text {th }}$ and $j^{\text {th }}$ antennas, $t_{i j}^{\prime}$ is estimated with timing error, $\sigma_{i j}$, then actual (primed) and measured (unprimed) quantities are related by

$$
\begin{aligned}
& \mathbf{r}_{i}^{\prime}=\mathbf{r}_{i}+\mathbf{e}_{i}, \\
& t_{i j}^{\prime}=t_{i j}+\sigma_{i j} .
\end{aligned}
$$

Solving (8) for $\mathbf{r}_{i}$ and $t_{i j}$, and using (2) and (3) gives,

$$
\mathbf{g}^{\prime}=\mathbf{g}+\boldsymbol{\delta}
$$

where the components of $\delta$ are given by

$$
\begin{aligned}
& \delta_{i j}=\mathbf{r}_{i} \cdot \mathbf{e}_{i}-\mathbf{r}_{j} \cdot \mathbf{e}_{j}+\frac{1}{2}\left(e_{i}^{2}-e_{j}^{2}\right) \\
& -v^{2}\left[t_{i 1} \sigma_{i 1}-t_{j} \sigma_{j 1}+\frac{1}{2}\left(\sigma_{i 1}^{2}-\sigma_{j}^{2}\right)\right]
\end{aligned}
$$

By similar means, we obtain an expression for the error-free kernel matrix, $\mathbf{K}^{\prime}$, in terms of the measured kernel,

$$
\mathbf{K}^{\prime}-\mathbf{K}+\mathbf{E}
$$

where the error matrix is given by,

$$
\mathbf{E}=\left[\begin{array}{cccc}
e_{x 21} & e_{y 21} & e_{z 21} & \sigma_{21} \\
e_{x 31} & e_{y 31} & e_{z 31} & \sigma_{31} \\
\vdots & \vdots & \vdots & \vdots \\
e_{x m 1} & e_{y m l} & e_{z m l} & \sigma_{m l}
\end{array}\right],
$$

and $e_{\alpha i j}=\left(\mathbf{e}_{i}-\mathbf{e}_{j}\right) \cdot \boldsymbol{\alpha}$ with $\boldsymbol{\alpha}$ denoting any of the coordinate unit vectors $\mathbf{x}, \mathbf{y}$, or $\mathbf{z}$.

Substituting (9) and (11) into (7) and inverting gives the retrieved solution, $f$, directly in terms of the actual source, $f^{\prime}$,

$$
\mathbf{f}=\mathbf{f}^{\prime}+\left(\mathbf{K}^{\mathbf{1}} \mathbf{K}\right)^{-1} \mathbf{K}^{\mathbf{1}}\left(\mathbf{E} \mathbf{f}^{\prime}-\boldsymbol{\delta}\right) .
$$

This result clarifies exactly how the retrieval of source location and activation time is distorted by measurement errors $(\boldsymbol{\delta}, \mathbf{E})$. Here, the errors $(\boldsymbol{\delta}, \mathbf{E})$ are related to the uncertainty in antenna placement, $\mathbf{e}_{i}$, and time difference error, $\sigma_{i j}$, as provided in (10) and (12), respectively. Note that the retrieval error, $\left.\varepsilon \equiv \mathbf{K}^{t} \mathbf{K}\right)^{-1} \mathbf{K}^{\mathrm{t}}\left(\mathbf{E} \mathbf{f}^{\prime}-\boldsymbol{\delta}\right)$ is proportional to the actual source, $\mathbf{f}^{\prime}$. This implies that distant sources (those with large values of $x^{\prime}, y^{\prime}$, and/or $z^{\prime}$ ) are more difficult to retrieve accurately.

The result in (13) also clarifies how network geometry and source location amplify, or otherwise distort, the measurement errors. The minimum eigenvalue of $\mathbf{K}^{\mathbf{t}} \mathbf{K}$ (and hence the degree of retrieval error magnification) can be modulated by adjusting the antenna network geometry and source location. If the minimum eigenvalue of $K^{\prime} K$ is small, large elements will appear in $\left(\mathbf{K}^{\mathbf{K}}\right)^{-1}$, making the retrieval error, $\mathbf{E}$, large. In addition, the minimum eigenvalue of $K^{\mathbf{t}} \mathbf{K}$ can also be increased or decreased simply by changing the differencing scheme described in section 2 immediately following (5). The reader is referred to Twomey [1977; chapter 6] for more on the topic of error magnification by small eigenvalues.

\section{Antennas in the Plane}

If five antennas are placed on flat terrain (vertically adjustable antenna mounts could be employed if terrain is not sufficiently

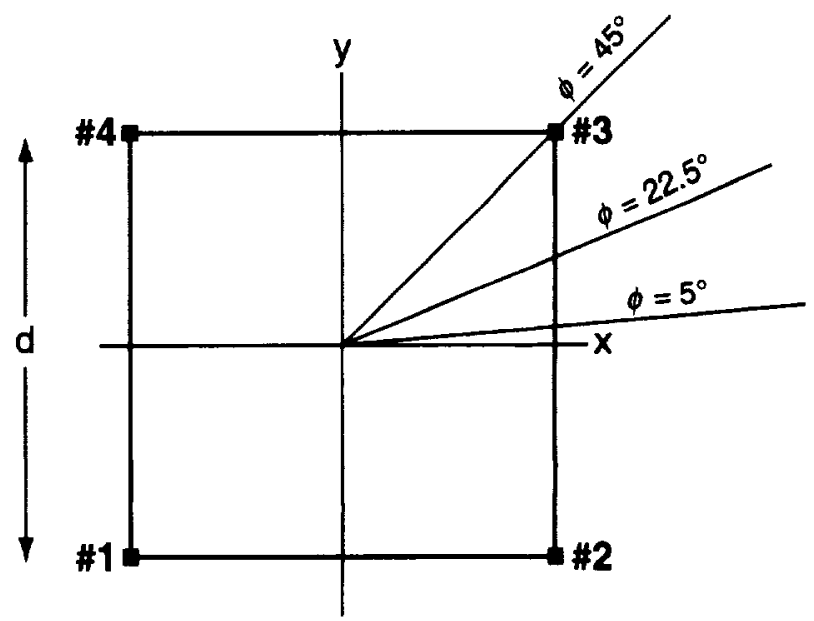

Figure 2. Square antenna network geometry, and the three azimuths along which simulated lightning radio sources were placed. 
flat), the third column of $\mathbf{K}$ will be all zeroes. In this case, the third column and fourth row of $\mathbf{K}$ can be eliminated, resulting in a $(3 \times 3) \mathbf{K}$-matrix. Thus, if just four antennas are located in a plane, the solution becomes:

$$
\mathbf{f}=\left[\begin{array}{c}
x \\
y \\
-v^{2} t
\end{array}\right]=\left[\begin{array}{ccc}
x_{21} & y_{21} & t_{21} \\
x_{31} & y_{31} & t_{31} \\
x_{41} & y_{41} & t_{41}
\end{array}\right]^{-1}\left[\begin{array}{l}
g_{21} \\
g_{31} \\
g_{41}
\end{array}\right]=\mathbf{K}^{-1} \mathbf{g}
$$

and the altitude is obtained by back substitution into (1), that is, $z$ $=\left[v^{2}\left(t_{1}-t\right)^{2}-\left(x_{1}-x\right)^{2}-\left(y_{1}-y\right)^{2}\right]^{1 / 2}$. In the following sections we test the linear theory by considering a square network and a triangular network, each composed of four antennas.

\section{Square Network Results}

If four antennas are placed in a square configuration with baseline $d$ as shown in Figure 2, the analytic forms of $\mathbf{K}$ and $\mathbf{K}^{-1}$ become,

$$
\begin{gathered}
\mathbf{K}=\left[\begin{array}{ccc}
-d & 0 & t_{12} \\
0 & -d & t_{23} \\
d & 0 & t_{34}
\end{array}\right], \\
\mathbf{K}^{-1}=\frac{1}{d\left(t_{12}+t_{34}\right)}\left[\begin{array}{ccc}
-t_{34} & 0 & t_{12} \\
t_{23} & -\left(t_{12}+t_{34}\right) & t_{23} \\
d & 0 & d
\end{array}\right],
\end{gathered}
$$

and we have used the differencing scheme (site 1 - site 2 , site 2 site 3 , site 3 - site 4 \}. Note that $K$ is singular whenever $\left(t_{12}+t_{34}\right)$ $=0$, that is, whenever a source is located on or above the $x$ or $y$ axes. Hence, as a source is moved progressively closer to these symmetry axes, the minimum eigenvalue of $\mathbf{K}$ approaches zero, the elements of $\mathbf{K}^{-1}$ become very large, and the error vector $\left(\mathbf{E} \mathbf{f}^{\prime}-\boldsymbol{\delta}\right.$ ) in (13) becomes excessively magnified. That is, the retrieval error, $\varepsilon$, grows without bound. In effect, the network becomes progressively "blind" to sources located near the symmetry axes. (Note that for a determined system, (13) reduces to: $f=f^{\prime}+K^{-1}\left(E f^{\prime}-\delta\right)$.

In our computer-simulated retrieval of known radio sources, we placed the sources along azimuths $=5^{\circ}, 22.5^{\circ}$, and $45^{\circ}$ as shown in Figure 2 and varied the distance, $\rho$, from the center of the network. The locations chosen along these azimuths were $500 \mathrm{~m}$ apart. Time difference and antenna location errors were randomly varied from 0 to $50 \mathrm{~ns}$ and 0 to $10 \mathrm{~cm}$, respectively, and 100 retrievals were performed at each location.

Mean spatial and temporal retrieval errors are provided in Figure 3 for sources with altitudes of $7 \mathrm{~km}$. Note that the errors (and the fluctuation of the errors) increase for sources near the $x$ and $y$ axes as predicted above from the form of $\mathbf{K}^{-1}$. Note also that the errors increase for distant sources as predicted by (13). Similar retrieval errors are obtained when the known source altitude is lowered to $2 \mathrm{~km}$ or raised to $10 \mathrm{~km}$. Of course, our simulations do not account for ground reflections/impedences that may occur in nature for low elevation sources.

\section{Triangular Network Results}

It is natural at this point to ask the question: Is it possible to select a specific network geometry that is not "blind" to any

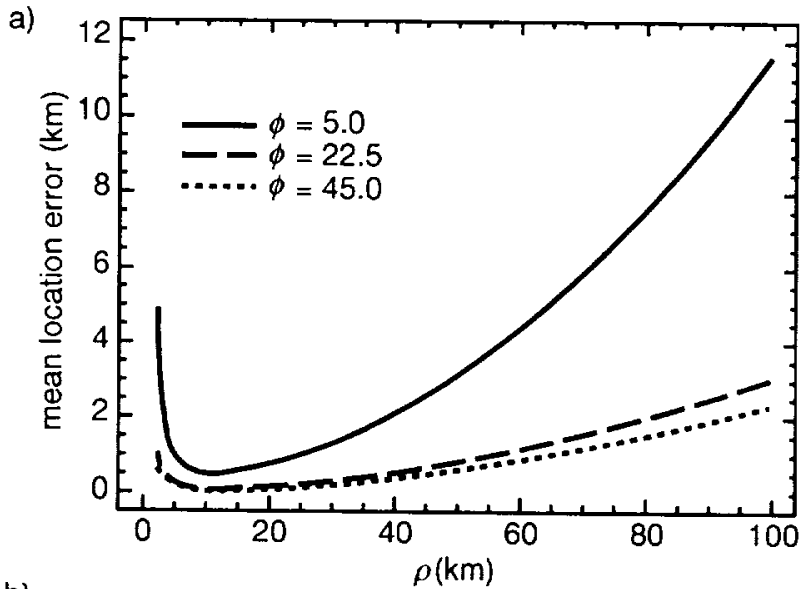

b)

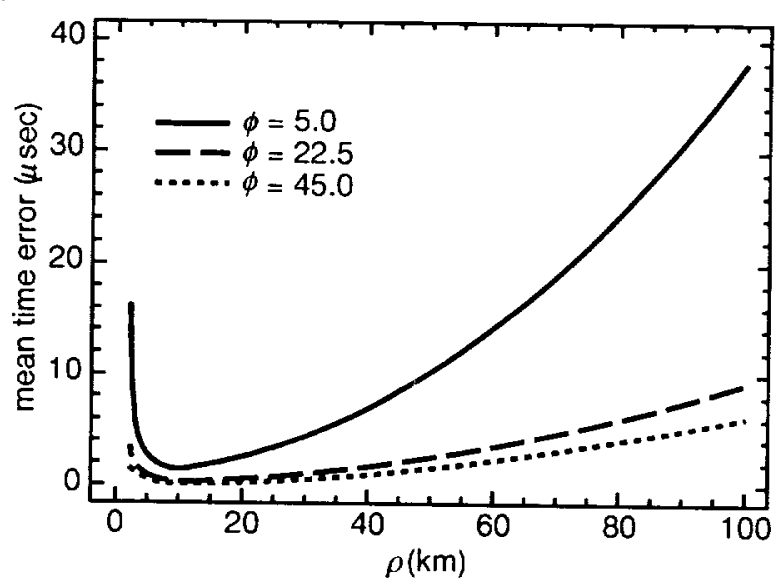

c)

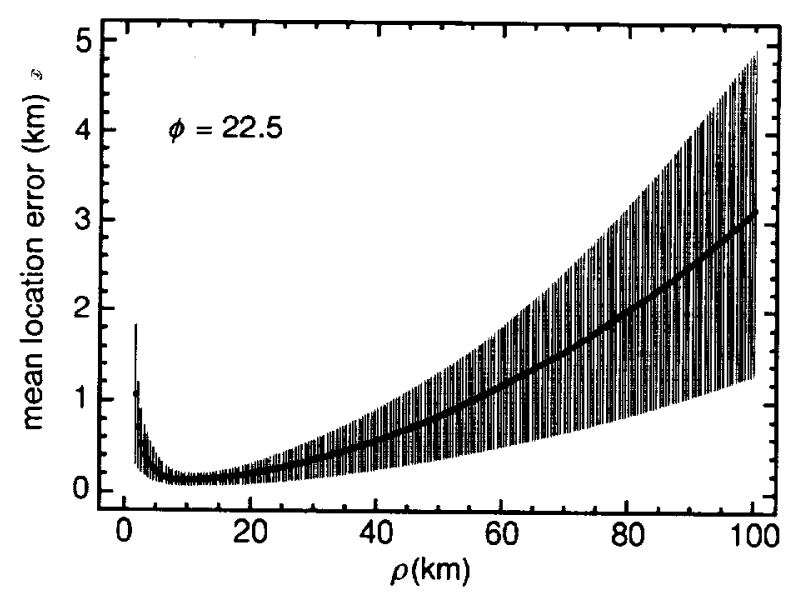

Figure 3. Mean (a) location and (b) time-of-occurrence error for sources placed in the vicinity of the square network along the azimuths shown in Figure 2. Source altitude is $7 \mathrm{~km}$. Figure $3 \mathrm{c}$ shows the mean and standard deviation (vertical lines) for the case $\phi=22.5^{\circ}$.

source location? Mathematically, this is equivalent to asking whether or not there exists a network geometry such that $\mathbf{K}$ is nonsingular for all source locations $(x, y, z)$. We begin by considering a triangular network geometry given in Figure 4; this geometry has been referred to as a "Y" configuration by Poehler [1977; 1979] as is evident if Figure 4 is inverted. Three of the four antennas are placed at the vertices of an equilateral triangle, and the fourth is placed at the center of the triangle. The distance from the central antenna (taken as the origin) to any of the other 


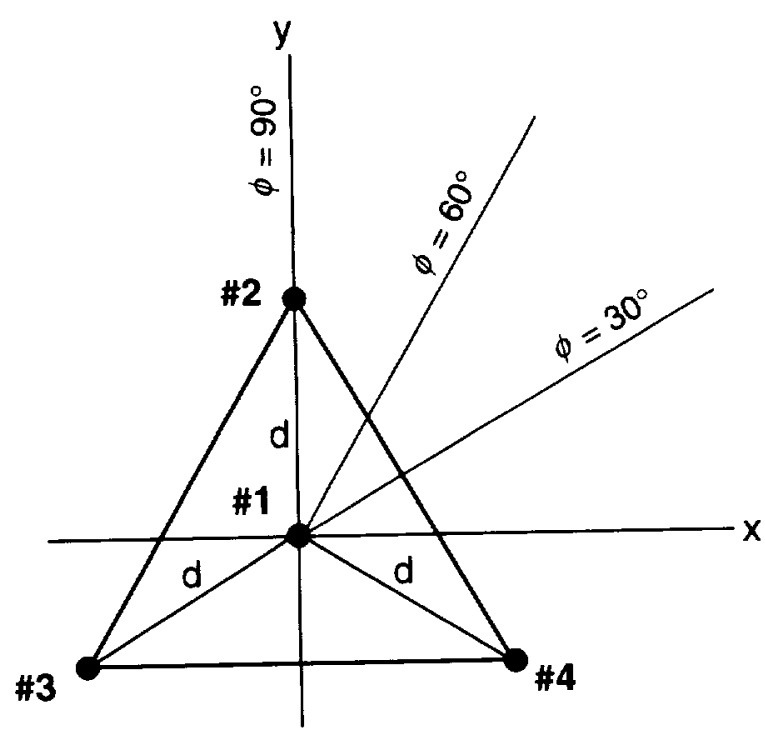

Figure 4. Triangular network geometry, and the three azimuths along which simulated lightning radio sources were placed.

antennas is given by $d$. An arbitrary source location is a distance $r=\left(x^{2}+y^{2}+z^{2}\right)^{1 / 2}$ from the central site, and the distance between the source and the remaining antennas is the magnitude of the relative position vector, $R_{i}=\left[\left(x_{i}-x\right)^{2}+\left(y_{i}-y\right)^{2}+z^{2}\right]^{1 / 2}$.

For this network, the analytic forms for $\mathbf{K}$ and $\mathbf{K}^{-1}$ are

$$
\begin{aligned}
& \mathbf{K}=\left[\begin{array}{ccc}
0 & -d & t_{12} \\
d \cos \frac{\pi}{6} & d\left(1+\sin \frac{\pi}{6}\right) & t_{23} \\
-2 d \cos \frac{\pi}{6} & 0 & t_{34}
\end{array}\right] \text {, } \\
& \mathbf{K}^{-1}=\frac{-1}{t_{21}+t_{31}+t_{41}}\left[\begin{array}{ccc}
\frac{3 t_{34}}{2 d \cos \frac{\pi}{6}} & \frac{t_{34}}{d \cos \frac{\pi}{6}} & \frac{-\left(3 t_{12} / 2+t_{23}\right)}{d \cos \frac{\pi}{6}} \\
\frac{-\left(2 t_{23}+t_{34}\right)}{d} & \frac{2 t_{12}}{d} & \frac{t_{12}}{d} \\
3 & 2 & 1
\end{array}\right.
\end{aligned}
$$

where we have used the differencing scheme given in section 6 and we have repetitively applied (6) to simplify the form of $\mathbf{K}^{-1}$. If we change the differencing scheme to that implied by (5), that is: \{site 2 - site 1 , site 3 - site 1 , site 4 - site 1\}, we obtain:

$$
\mathbf{K}=\left[\begin{array}{ccc}
0 & d & t_{21} \\
-d \cos \frac{\pi}{6} & -d \sin \frac{\pi}{6} & t_{31} \\
d \cos \frac{\pi}{6} & -d \sin \frac{\pi}{6} & t_{41}
\end{array}\right]
$$

$$
\mathbf{K}^{-1}=\frac{1}{t_{21}+t_{31}+t_{41}}\left[\begin{array}{ccc}
\frac{t_{31}-t_{41}}{2 d \cos \frac{\pi}{6}} & \frac{-\left(t_{21} / 2+t_{41}\right)}{d \cos \frac{\pi}{6}} & \frac{\left(t_{21} / 2+t_{31}\right)}{d \cos \frac{\pi}{6}} \\
\frac{t_{31}+t_{41}}{d} & \frac{-t_{21}}{d} & \frac{-t_{21}}{d} \\
1 & 1 & 1
\end{array}\right]
$$

Since $t_{i j}=\left(R_{i}-R_{j}\right) / v$, it follows that $\mathbf{K}$ (in both (16) and (17)) is singular if and only if $r$ is the average of $\left\{R_{2}, R_{3}, R_{4}\right\}$. We have scanned a large $(x, y, z)$ grid volume with a computer to evaluate $r, R_{2}, R_{3}$, and $R_{4}$ and have found no singularities in $\mathbf{K}$. However, to the best of our knowledge the nonsingular nature of $\mathbf{K}$ has not been rigorously proven. A tedious, yet complete proof is provided in the appendix.

The results of the ismputer simulation for the triangular network geometry are given in Figure 5 for the two differencing schemes provided in (16) and (17). As seen in Figure 4, we have chosen three symmetry azimuths $\phi=30^{\circ}, 60^{\circ}$, and $90^{\circ}$ to charac terize the errors throughout the $x y$ plane. Once again, we see errors increase with distance from the network as predicted by (13). What is most interesting to note from Figure 5 is that the differencing scheme used in (17) provides errors that are almost

a)

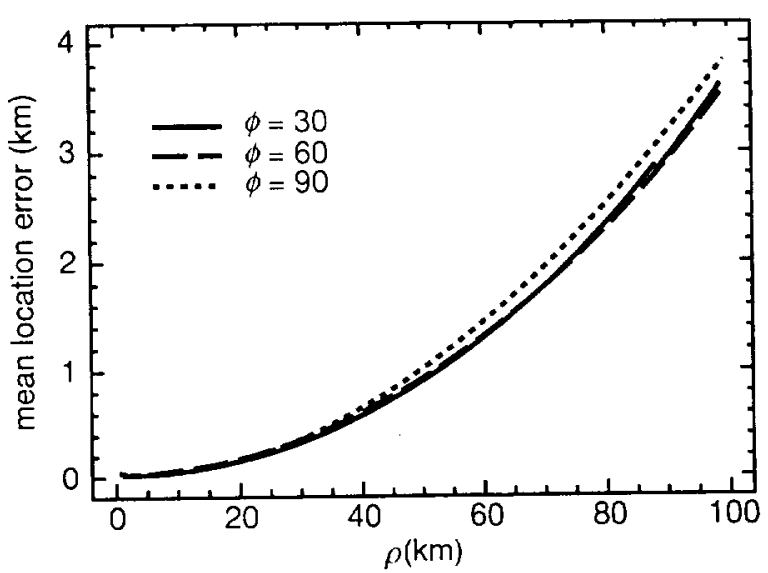

b)

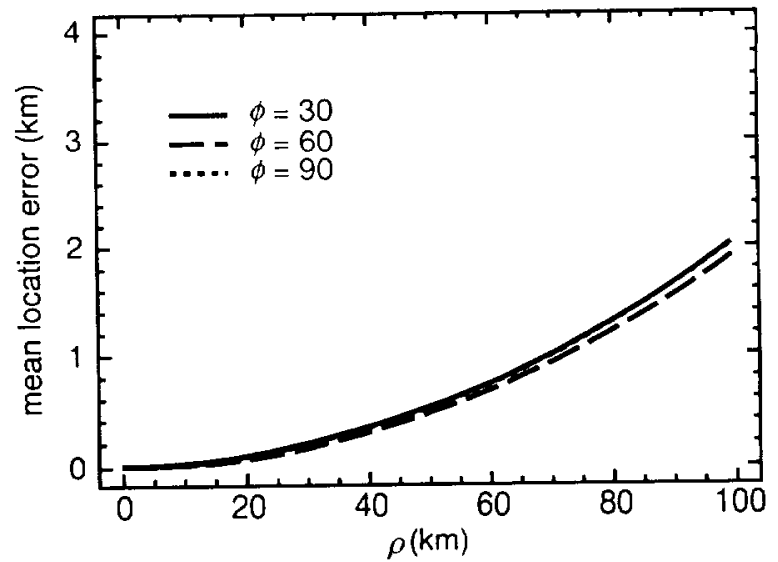

Figure 5. Mean location error for sources placed in the vicinity of the triangular network along the azimuths shown in Figure 4 for antenna differencing scheme (a) $\{1-2,2-3,3-4\}$ (b) $\{2-1$, $3-1,4-1\}$; where for example, site $1=1$. Source altitude is $7 \mathrm{~km}$. 
$50 \%$ less (at $100 \mathrm{~km}$ ) than those errors obtained using the differencing scheme in (16).

\section{Seven-Antenna Network Results}

The NASA-KSC LDAR system recently discussed by Maier et al. [1995] has a total of seven ground-based antennas for determining the sources of VHF pulses at $66 \mathrm{MHz}$. The network has one central site and six antennas that are separated in azimuth by roughly $60^{\circ}$. The six outer sites are nearly equidistant from the central site. Hence, the LDAR network can approximately be viewed as two separate triangular networks (see Figure 4) that are rotated by $180^{\circ}$ from one another and that share the same central site. The source location is found by inverting a determined linear system that is similar, but not identical to, the system given in (14) for one of the triangular networks. A solution is also found for the other $\left(180^{\circ}\right.$ rotated) triangular network. The two solutions are compared and if consistent a final answer is given. If the solutions are not consistent, 18 additional four-antenna network inversions are performed. If sufficient consistency is apparent, a final solution based on the 20 separate solutions (each appropriately weighted) is obtained (i.e., the central site is used in each derived four antenna network so there are a combination of six antennas taken three at a time, or a total of 20 possible derived networks). Additional details are provided by Maier et al. [1995].

Instead of performing multiple retrievals to obtain a final solution, we perform one inversion of the overdetermined system given in (3) with $m=6$. The seven-antenna network that we use is that of two triangular networks that are rotated $180^{\circ}$ apart from one another and that share the same central site. The error results for two symmetry azimuths are provided in Figure 6. It is clear from this result that the single overdetermined retrieval is more accurate than any of the previous four antenna network results given above. Furthermore, a single retrieval generally requires less computer CPU time than multiple retrievals (and solution comparisons) performed with the current LDAR system.

Nonetheless, given high-speed computers of today, CPU time expenditure is not a problem with the current LDAR algorithm. Indeed, both the LDAR algorithm and the solutions we have presented do not require actual "matrix inversions," but are closed form forward computations based on analytic expressions for the matrix inverse.

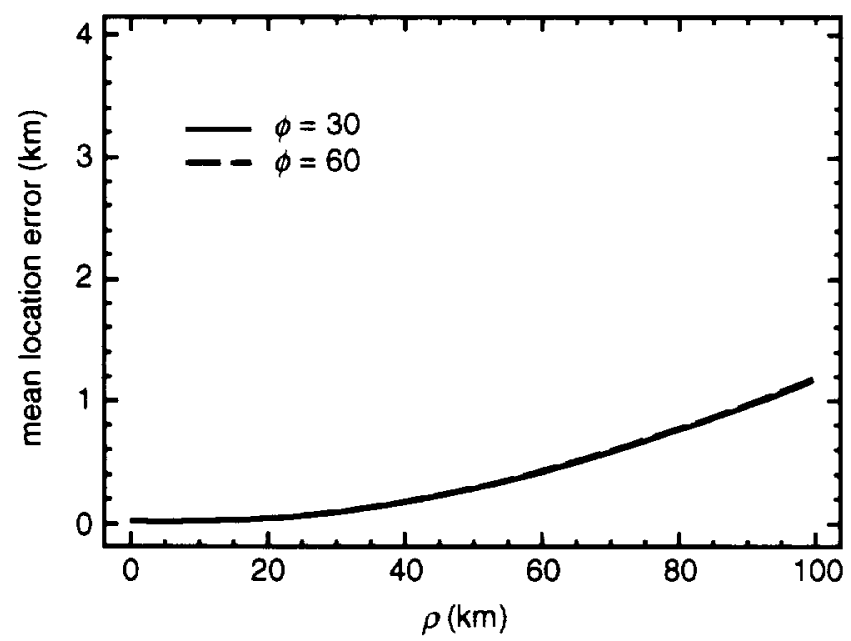

Figure 6. Mean location error for a symmetric seven antenna network with all sites differenced with respect to the central site. Source altitude is $7 \mathrm{~km}$.
In addition, there is a benefit in computing several solutions in the current LDAR algorithm. The solution comparison process described in Maier et al. [1995] helps "weed-out" poor solutions (e.g., as might be obtained when the network triggers on two or more distinct VHF sources).

However, in the case of seven-station retrievals that give poor (i.e., nonphysical) results, we suggest removing one site and then performing a six-station retrieval. If there is no improvement in the solution, a different site can be removed so that an alternate six-station retrieval can be completed. One can permute through all such possibilities. If there is still no improvement, two sites can be removed, etc., or even three sites.

In effect, we do not force a four-antenna retrieval. Instead, we take full advantage of an overdetermined retrieval whenever possible. In this way, we minimize retrieval error.

\section{Summary}

We have reexamined the problem of retrieving radio source location and time-of-occurrence from TOA data. We have suggested the more appropriate terminology for a TOA measurement network as a "hyperplane system," since the source location and time-of-occurrence is most concisely viewed as a geometrical intersection of hyperplanes in Minkowski space. With this formalism, we have derived an analytic expression for retrieval error as given in (13) that enhances earlier nonlinear GDOP error analyses provided by Holmes and Reedy [1951]. In so doing, we have clearly identified the important variables that affect retrieval accuracy: network geometry, number of antennas, differencing scheme, source location, timing error, and antenna location error. Computer simulations have been added to help elucidate and confirm the attributes/predictions of the retrieval equation in (13).

In the future, we intend to apply our linear inversion analysis to actual LDAR TOA data derived from Florida thunderstorms. We will attempt to make further comparisons between the retrieval algorithm currently in use with LDAR and our algorithm. As part of algorithm optimization, we shall also investigate several external constraints to the solution process that, based on real data retrievals and computer simulations, prove to stabilize the final solution. Finally, one author shall investigate the possibility of using LDAR retrievals to help constrain the location of lightning charges as derived from the Advanced Ground Based Field Mill (AGBFM) network at NASA-KSC.

\section{Appendix: The Nonsingular Nature of $K$ for the Triangular Network}

Here, we show that the $(3 \times 3)$ kernel matrix, $\mathbf{K}$, for the triangular network (with any nondegenerate differencing scheme, for example, as in (16) or (17)) is nonsingular. This is equivalent to showing that the average of the distances from an arbitrary point to the three vertices of an equilateral triangle is greater than the distance from that point to the centroid. The desired result is obtained by seeking the extrema of the sum of distances from the vertices to a point on a hemisphere of radius $r$ centered at the centroid of the triangle. Full generality is recovered by allowing $r$ to be arbitrary. We set up a Cartesian coordinate system, with points specified by $(x, y, z)$, and its origin at the centroid. Vertices are located at

$$
\mathbf{d}_{2}=d \hat{\mathbf{y}}, \mathbf{d}_{3}=-d\left(\frac{\sqrt{3}}{2} \hat{\mathbf{x}}+\frac{1}{2} \hat{\mathbf{y}}\right), \mathbf{d}_{4}=d\left(\frac{\sqrt{3}}{2} \hat{\mathbf{x}}-\frac{1}{2} \hat{\mathbf{y}}\right)
$$

the distance from the centroid to a vertex is $d$. For the present, 
assume $r \geq d$. It will be convenient to consider the distances from the vertices on an orthogonal coordinate system. We will set $R_{2}=$ $X, R_{3}=Y$, and $R_{4}=Z$. Vectors from antennas $I$ through 4 to the source will be denoted by $\mathbf{r}, \mathbf{X}, \mathbf{Y}, \mathbf{Z}$, respectively. Let $\boldsymbol{\theta}$ denote the angle between $\mathbf{d}_{2}$ and $\mathbf{r}$. Employing the law of cosines, it may be shown that

$$
3 r^{2}=X^{2}+Y^{2}+Z^{2}-3 d^{2}
$$

It will be necessary to extremize

$$
F(X, Y, Z)=X+Y+Z
$$

subject to (A2).

Using Lagrange multipliers or any other suitable method, the only extremum in the first octant is $X=Y=Z=\sqrt{r^{2}+d^{2}} ; F=$ $3 \sqrt{r^{2}+d^{2}}>3 r$. This turns out to be a local maximum. Minima must be found by looking along boundaries. These are not necessarily in any coordinate plane. All of $X, Y, Z$ must be greater than or equal to $r-d$. Eliminating $Z$ reduces the problem to finding the minima of:

$$
\begin{gathered}
f(X, Y)=X+Y+Z(X, Y), \\
Z(X, Y)=\sqrt{3\left(r^{2}+d^{2}\right)-X^{2}-Y^{2}}
\end{gathered}
$$

with the requirement that none of $X, Y, Z$ be negative. An addi tional constraint is obtained from (A2),

$$
X^{2}+Y^{2} \leq 2\left(r^{2}+d^{2}+r d\right) \text {. }
$$

This constraint does not sufficiently limit $f ; X$ and $Y$ cannot both attain their minimum values of $r-d$ at the same time. Boundaries are shown in Figure A 1 . The smallest values of $Y$ for a fixed $X$ occur when $z=0$ (the lightning event is in the plane of the triangle). This may be demonstrated by writing

$$
Y^{2}=r^{2}+d^{2}-2 \mathbf{r} \cdot \mathbf{d}_{3}
$$

Elevating the lightning event from the plane of the triangle while keeping $X$ and $r$ constant is equivalent to rotating $\mathbf{X}$ and $\mathbf{r}$ about $\mathbf{d}_{2}$. Here, $\mathbf{d}_{3}$ has no $z$ component and $y$ is constant. The value of $Y$ is minimized when $x$ is as small as possible (at $z=0$ ).

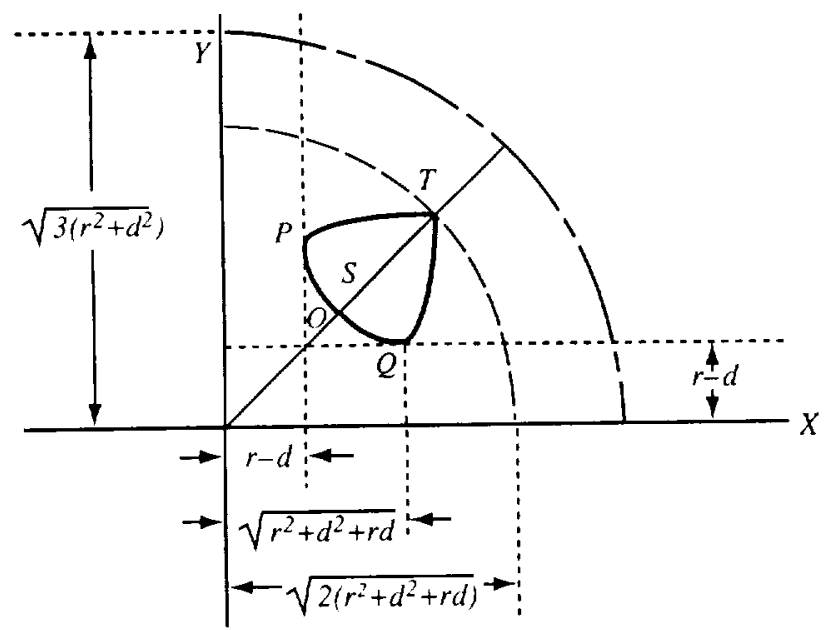

Figure A1. Boundaries in the $X Y$ plane.
The slope of a tangent to $\overline{P O Q}$ is given by

$$
\frac{d Y}{d X}=-\frac{\sin (2 \pi / 3-\theta)}{\sin \theta} \frac{X}{Y} \text {. }
$$

It is never positive and goes from 0 at $\left(\sqrt{r^{2}+d^{2}+r d}, r-d\right)$ to $-\infty$ at $\left(r-d, \sqrt{r^{2}+d^{2}+r d}\right)$. At the point $O, X=Y, \theta=\pi / 3$, and $d Y / d X=-1$. The coordinates of this point are $X=Y=$ $\sqrt{r^{2}+d^{2}-r d}$.

The gradient

$$
\nabla f(X, Y)=(1-X / Z) \hat{\mathbf{X}}+(1-Y / Z) \hat{\mathbf{Y}}
$$

points in the direction of greatest increase of $f$. The region in Figure $\mathrm{Al}$ can be thought of as a mountain with its summit at $X=$ $Y=\sqrt{r^{2}+d^{2}}$ (the point labeled $S$ ). On the square region $0 \leq X \leq \sqrt{r^{2}+d^{2}}, 0 \leq Y \leq \sqrt{r^{2}+d^{2}}$, we have $Z \geq X$ and $Z \geq Y$. Except at $S$, the inequality is strict. The gradient points up and to the right. Below the line segment $\overline{O S}, \pi / 3 \leq \theta \leq 2 \pi / 3$, and $X>$ $Y$. The opposite is true above $\overline{O S}$. The mountain has a ridge along this line segment. Below and to the left of $S$, it is clear that as $X$ and $Y$ decrease, $Z$ increases (the components of the gradient remain positive). Values of $f$ beneath or to the left of $\overline{P O Q}$ are smaller than values on this curve.

It will not be necessary to investigate the values of $f$ along $\overline{P T}$ or $\overline{Q T}$. These curves can be mapped onto $\overline{P O Q}$ by a suitable relabeling of $\mathbf{X}, \mathbf{Y}, \mathbf{Z}$. Symmetry may be further exploited to reduce the effort. An interchange of $\mathbf{X}$ and $\mathbf{Y}$ will map $\overline{O P}$ and $\overline{O Q}$ onto each other.

It tums out to be difficult to parameterize $Y$ in terms of $X$ and obtain the minima along the bounding curve by differentiation. We will bound the values of $f$ along $\overline{O Q}$ by those on a simpler polygonal boundary below $\overline{O Q}$.

We begin at $Y=r-d$ and require that

$$
f(X, r-d)=X+(r-d)+Z(X, r-d) \geq 3 r .
$$

This leads to the inequalities

$$
\begin{gathered}
2 X^{2}-2(2 r+d) X+2 r^{2}-d^{2}+2 r d \leq 0, \\
r-\frac{\sqrt{3}-1}{2} d \leq X \leq r+\frac{\sqrt{3}+1}{2} d .
\end{gathered}
$$

The largest value of $X$ that needs to be considered is $\sqrt{r^{2}+d^{2}+r d}$. Extrema along this line segment, denoted by $\overline{Q B}$ (see Figure $A 2$ ), must be at the endpoints

$$
\begin{gathered}
f\left(\sqrt{r^{2}+d^{2}+r d}, r-d\right)=r-d+2 \sqrt{r^{2}+d^{2}+r d}>3 r, \\
f\left(r-\frac{\sqrt{3}-1}{2} d, r-d\right)=3 r .
\end{gathered}
$$

The next portion of the bounding curve is drawn by leaving $X=r-(\sqrt{3}-1) d / 2$ and increasing $Y$ to some point below $\overline{O Q}$. 


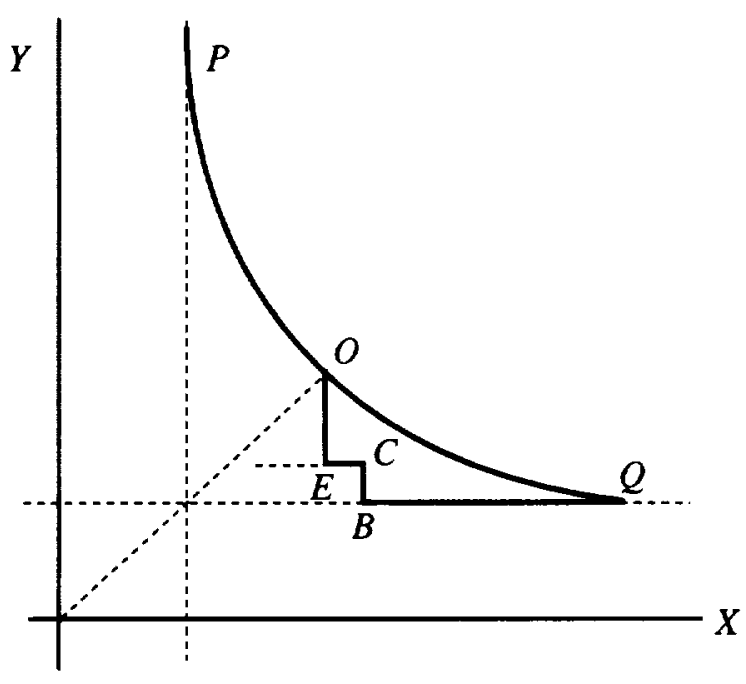

Figure A2. Polygonal path.

The location of this point may be found by solving for $\cos \theta$ in $X^{2}=r^{2}+d^{2}-2 r d \cos \theta$. We find that

$$
\cos \theta=\frac{\sqrt{3}-1}{2}+\frac{\sqrt{3}}{4} \frac{d}{r}
$$

$$
\begin{aligned}
& \cos \left(\frac{2 \pi}{3}-\theta\right)=\frac{\sqrt{3}}{2} \sqrt{1-\left[\frac{\sqrt{3}-1}{2}+\frac{\sqrt{3 d}}{4 r}\right]^{2}} \\
& -\frac{1}{2}\left[\frac{\sqrt{3}-1}{2}+\frac{\sqrt{3} d}{4 r}\right] \leq \frac{3^{3 / 4}}{2^{3 / 2}}-\frac{\sqrt{3}-1}{4}<\frac{2}{3} .
\end{aligned}
$$

Using the law of cosines to write $Y^{2}$ in terms of $r, d$, and $\cos (2 \pi / 3-\theta)$, we see that $Y$ decreases as $\cos (2 \pi / 3-\theta)$ increases. The smallest value of $Y$ on $\overline{P O Q}$ for $X=r-(\sqrt{3}-1) d / 2$ is bounded using (A12);

$$
Y \geq \sqrt{r^{2}+d^{2}-4 r d / 3}>r-2 d / 3 .
$$

We can proceed up to point $C$, whose coordinates are $(r-(\sqrt{3}-1) d / 2, r-2 d / 3)$.

As for the first part of the polygonal bounding curve, the extrema are at the endpoints. The minimum value of $f$ along $\overline{B C}$ is $3 r$ and occurs at $B$. The maximum is located at $C$ and is given by

$$
\begin{aligned}
& 2 r-[(\sqrt{3}-1) / 2+2 / 3] d \\
& +\sqrt{r^{2}+(14 / 9+\sqrt{3} / 2) d^{2}+(\sqrt{3}+1 / 3) r d}>3 r
\end{aligned}
$$

Proceeding as before, we fix $Y=r-2 d / 3$ and see how far to the left we can go and still have $f \geq 3 r$. This time we obtain the inequality

$$
r-\frac{\sqrt{42} / 2-1}{3} d \leq X \leq r+\frac{\sqrt{42} / 2+1}{3} d,
$$

It will not be necessary to proceed from $C$ all the way to the value indicated in (A15). We can stop at a point $E$ which has the same $X$-coordinate as $O$. From the estimate

$$
\left(r-\frac{\sqrt{42} / 2-1}{3} d\right)^{2}<(r-0.7 d)^{2}<r^{2}+d^{2}-r d,
$$

we see that $f(X, Y)>3 r$ at every point of this portion of the boundary.

Finally, we proceed vertically from $E$ to $O$. The derivative of $f$ with respect to $Y$ is positive for $Y$ below $O$. The smallest value of $f$ here is at $E$.

Because of the relative positions of the actual and polygonal boundaries and the topography of the surface, the inequality is strict; $X+Y+Z>3 r$. When $r>0$, the result for the case $r \leq d$ may be inferred by interchanging $r$ and $d$ in the preceding calcu lations and showing that $X+Y+Z>3 d \geq 3 r$. The case $r=0$ is immediate.

When $r \gg d$, the order of magnitude of the eigenvalues may be found. In this case the vectors $X, Y, Z$, and $\mathbf{r}$ are all almost parallel. Consider two parallel planes whose common normal is in the same direction as $\mathbf{r}$. One is located at the origin, the other passes through the source point. The magnitude of $\mathbf{X}$ can be written as

$$
X^{2}=X^{\prime 2}+\left|\mathbf{d}_{2} \times \hat{\mathbf{r}}\right|^{2}
$$

where $X^{\prime}$ is the distance from the head of $\mathbf{d}_{2}$ to the plane at the source point. Similar relations hold for $Y$ and $Z$.

The magnitudes of $\mathbf{X}^{\prime}, \mathbf{Y}^{\prime}, \mathbf{Z}^{\prime}$ may be found by subtracting the dot products $\mathbf{d}_{2} \cdot \hat{\mathbf{r}}, \mathbf{d}_{3} \cdot \hat{\mathbf{r}}, \mathbf{d}_{4} \cdot \hat{\mathbf{r}}$ from $X, Y, Z$ respectively. Setting $\mathbf{d}_{j} \cdot \hat{\mathbf{r}}=m_{j}, j=2,3,4$, we find

$$
\begin{aligned}
m_{2}=\frac{d}{r} y, m_{3} & =-\frac{d}{r}\left(\frac{\sqrt{3}}{2} x+\frac{y}{2}\right), m_{3}=\frac{d}{4}\left(\frac{\sqrt{3}}{2} x-\frac{y}{2}\right) \\
\sum_{j=2}^{4} m_{j} & =0
\end{aligned}
$$

The leading approximation $X=X^{\prime}$, etc., is not sharp enough in this regard. A second-order approximation is needed.

Let $\left|\mathbf{d}_{j} \times \hat{\mathbf{r}}\right|=n_{j}$. The length $X$ may be written in the form

$$
\begin{aligned}
X & =\sqrt{X^{\prime 2}+n_{2}{ }^{2}}=\sqrt{\left(r-m_{2}\right)^{2}+n_{2}{ }^{2}} \\
& =r-m_{2}+\frac{n_{2}{ }^{2}}{2 r}+O\left(r^{-2}\right)
\end{aligned}
$$

Summing (A19) with similar expressions for $Y$ and $Z$ gives

$$
X+Y+Z \approx 3 r+\frac{n_{2}^{2}+n_{3}^{2}+n_{4}{ }^{2}}{2 r} .
$$

Since not all of the $n_{j}$ 's can be zero at the same time, $X+Y+Z$ $>3 r$. Substituting for $\mathbf{d}_{j}$ and $\mathbf{r}$ yields 


$$
n_{2}^{2}+n_{3}^{2}+n_{4}^{2}=\frac{3 d^{2}}{2 r^{2}}\left(x^{2}+y^{2}+2 z^{2}\right)
$$

from which we see that $t_{21}+t_{31}+t_{41}=O\left(\frac{d^{2}}{v r}\right)$.

Acknowledgments. We appreciate the work of Susan Burrer, Shelby Morris, and Janine Roskowski for their efforts in organizing the final manuscript. We are also indebted to Carl Lennon and Launa Maier of the NASA-KSC for their many insightful comments regarding the LDAR system, and Hugh Christian of the NASA-MSFC for his support and encouragement in the early phase of this research.

\section{References}

Bent, R. B., P. W. Casper, T. H. Scheffler, and R. Leep, A unique time of arrival technique for accurately locating lightning over large areas. Preprints, Fifith Symposium on Meteorological Observations and Instrumentation, April 11-15, Toronto, Ontario, Canada, American Meteorological Society, Boston, 505-511, 1983.

Cianos, N., G. N. Oetzel, and E. T. Pierce, A technique for accurately locating lightning at close ranges, J. Appl. Meteorol., 11, 1120-1127, 1972.

Hager, W. H., and D. Wang, An analysis of errors in the location, current, and velocity of lightning, J. Geophys. Res., 100, 25, 721-25,729, 1995.

Holle, R. L., and R. E. Lopez, Overview of real-time lightning detection systems and their meteorological uses, NOAA Tech. Mem. ERL NSSL-102, 1993.

Holmes, T. G., and P. H. Reedy, Geometrical Dilution of Precision, Air Force Tech. Rep. 2l, Air Force Missile Test Cen., Patrick Air Force Base, Florida, 1951.

Koshak, W. J., and H. J. Christian, A nonhyperbolic solution to the problem of mapping lightning sources using VHF time-of-arrival measurements, 1994, Eos, Trans. AGU, 75(44), 104, Fall Meet. Suppl. 1994.

Lewis, E. A., R. B. Harvey, and J. E. Rasmussen, Hyperbolic direction finding with sferics of transatlantic origin, J. Geophys. Res., 65, 18791905. 1960.
MacClement, W. D., and R. C. Murty, VHF direction finder studies of lightning, J. Appl. Meteorol., 17, 786-795, 1978.

Maier, L. M., C. L. Lennon,T. Britt, and S. Schaefer, Lightning detection and ranging (LDAR) system performance analysis, in Proceedings of the 6th Conference of Aviation Weather Systems, AMS Meeting, January 15-20, Dallas, 1995.

Murty, R. C., and W. D. MacClement, VHF direction finder for lightning location, J. Appl. Meteorol., 12, 1401-1405, 1973.

Oetzel, G. N., and E. T. Pierce, VHF technique for locating lightning, Radio Sci., 4, 199-201, 1969.

Poehler, H. A., An Accuracy Analysis of the LDAR System, NASA Contract. Rep. CR-154631, 1977.

Poehler, H. A., Error Analysis of Y-Configured Hyperbolic and Alternative Systems, Tech. Rep. RCA 620-5006, 1979.

Poehler, H. A., and C. L. Lennon, Lightning Detection and Ranging System (LDAR) system description \& performance objectives, NASA Tech. Mem. TM-74105, 1979.

Proctor, D. E., A hyperbolic system for obtaining VHF radio pictures of lightning. J. Geophys. Res., 76, 1478-1 489, 1971.

Rustan, P. L., M. A. Uman, D. G. Childers, W. H. Beasley, and C. L. Lennon, Lightning source locations from VHF radiation data for a flash at Kennedy Space Center, J. Geophys. Res., 85, 4893-4903, 1980.

Taylor, W. L., A VHF technique for space-time mapping of lightning discharge processes, J. Geophys. Res., 83, 3575-3583, 1978.

Thomson, E. M., P. J. Medelius, and S. Davis, A system for locating the sources of wideband dE/dt from lightning, J. Geophys. Res., 99 , 22,793-22,802, 1994

Twomey, S. A., Introduction to the Mathematics of Inversion in Remote Sensing and Indirect Measurements, Elsevier, New York, 1977.

W. J. Koshak, Space Sciences Laboratory, NASA Marshall Space Flight Center, Huntsville, AL 35812. (e-mail: william.koshak @msfc.nasa.gov

R. J. Solakiewicz, Department of Mathematics and Computer Science, Chicago State University, Chicago, IL 60628-1598. (e-mail: bihkrso@vxa.ecn.logu.edu

(Received September 16, 1995; revised May 18, 1996; accepted May 21, 1996.) 
